\title{
Non-contact Measurement of Quality Factor for Monolithic Cylindrical Fused Silica Resonators
}

\author{
Dongya Wang ${ }^{a}$, Yao Pan ${ }^{b}$, Suyong Wu ${ }^{c^{*}}$, Hongchang Zhao, Tianliang Qu, Jianxun Tang* \\ Department of Opto-electronic Science and Engineering, National University of Defense \\ Technology, Changsha 410073, China
}

aemail:mywangdongya@126.com, bemail:panyao.liz@163.com, email:sywu2001@163.com

Keywords: non-contact measurement; cylindrical resonator; $Q$ factor; fused silica

\begin{abstract}
The monolithic cylindrical fused silica resonator is the critical component of a cylindrical resonator gyroscope, and the $\mathrm{Q}$ factor is the key parameter to evaluate the performance of a cylindrical resonator. In this paper, a non-contact measurement system based on acoustic excitation and laser Doppler detection was proposed to measure the $\mathrm{Q}$ factor of the cylindrical resonator. Two measurement methods (i.e. the frequency response method and the ring-down time method) were proposed, and the testing procedure was carefully designed. The reliability and stability of two methods were verified by simulations and experiments. Results showed that the ring-down time method can be applied to measure a wide range of $\mathrm{Q}$ factors with good repeatability, while the frequency response method is applicable only in low $\mathrm{Q}$ situations.
\end{abstract}

\section{Introduction}

Owing to the advantages of small size, light weight, low power consumption, long service life, the Coriolis vibratory gyroscope $(\mathrm{CVG})$ becomes increasingly widely used [1][2][3]. As an important kind of CVG, the cylindrical resonator gyroscope (CRG) has been intensively studied [4][5][6]. However, the resonators of the CVGs in these studies are made of metal, and the high damping of the material limits their $\mathrm{Q}$ factors to a low range. Therefore, we proposed a monolithic cylindrical resonator made from high purity fused silica [7]. This material is highly isotropic and has low internal friction; therefore it is preferred in high-Q resonators, such as cylindrical resonator gyroscopes, gravitational wave detectors, clocks, etc.

As the Q factor of the cylindrical fused silica resonator directly affects gyro performance, it is crucial to improve the resonator $\mathrm{Q}$ factor. To optimize the $\mathrm{Q}$ factor of the cylindrical resonators, it is essential to first accurately measure the $\mathrm{Q}$ factors. The traditional procedure to measure $\mathrm{Q}$ factor is using the amplitude frequency response characteristics of the cylindrical resonator excited by a sinusoidal sweeping signal. Some studies also use the ring-down time method to evaluate the damping properties of resonators [8]. In most $\mathrm{Q}$ factor measurements, the driving and sensing were realized by adding piezoelectric ceramics [9][10] or electrostatic capacitors [8][11]. However, piezoelectric ceramics must be bonded on the cylindrical resonator; therefore the measured Q factor includes the additional loss introduced by the glue and piezoelectric ceramics. Meanwhile, the electrostatic capacitors demand accurate positioning and the electronic circuits and the signal processing are relatively complicated.

In this paper, a Polytec PSV-500 laser Doppler vibrometer is used to build up a non-contact measurement system of $\mathrm{Q}$ factor for our cylindrical resonators. We first briefly introduced the measurement principles of both the frequency response method and the ring-down time method. Then we presented our measurement system and the testing procedures. We verified the measurement accuracy by comparing the experimental results with simulations. The reliability and stability of the measurement system was also demonstrated with experiments. Results showed that both methods can be well applied to measure low-Q resonators, and ring-down time method is preferred in measuring high-Q resonators. 


\section{Measurement principle}

Q factor of the resonator is defined as the total energy divided by energy loss per resonant cycle. It is inversely proportional to damping coefficient, therefore is commonly used to describe the damping "quality" of an oscillator.

$$
Q=2 \pi \frac{E_{\text {total }}}{\Delta E}=\frac{1}{2 \xi}
$$

where $E_{\text {total }}$ and $\Delta E$ are the total energy and energy loss per resonant cycle, respectively, and $\xi$ is the damping coefficient.

For a damped system, the forced vibration equation can be expressed as [12]

$$
\ddot{x}+2 \xi \omega_{0} \dot{x}+\omega_{0}^{2} x=\frac{f}{m} .
$$

where $x$ is the displacement of the mass, $\xi$ is the damping coefficient, $\omega_{0}$ is the angular frequency, $f$ is the external force, and $m$ is the effective mass.

The displacement frequency response function of the system is

$$
H_{d}(\omega)=\frac{1}{m\left(\omega_{0}^{2}+2 j \xi \omega_{0} \omega-\omega^{2}\right)} \text {. }
$$

By solving

$$
\frac{d\left|H_{d}(\omega)\right|}{d \omega}=0
$$

we can get

$$
\omega_{0}^{\prime}=\omega_{0} \sqrt{1-2 \xi^{2}},
$$

where $\omega_{0}^{\prime}$ represents the extreme value of displacement frequency response function. If $\xi \ll 0.1$, $\omega_{0}^{\prime}$ can be approximated as the resonant frequency $\omega_{0}$.

According to function (3) and (5), at half power point, the amplitude can be expressed as

$$
\frac{1}{\sqrt{2}}\left|H_{d}(\omega)\right|_{\max }=\frac{1}{m \sqrt{\left(\omega_{0}^{2}-\omega^{2}\right)^{2}+\left(2 \xi \omega_{0} \omega\right)^{2}}}=\frac{1}{\sqrt{2}} \frac{1}{2 m \xi \omega_{0}^{2} \sqrt{1-\xi^{2}}} .
$$

Then we can get

$$
\omega_{a, b}=\omega_{0} \sqrt{1-2 \xi^{2} \pm 2 \xi \sqrt{1-\xi^{2}}} .
$$

where $\omega_{a}$ and $\omega_{b}$ are the angular frequencies of the left and right half-power points, respectively.

If $\xi \ll 0.1$, then we have $\xi^{2} \approx 0$, therefore the quadratic terms of $\xi$ can be neglected

$$
\left\{\begin{array}{l}
\omega_{a} \approx \omega_{0} \sqrt{1-2 \xi} \approx \omega_{0}(1-\xi) \\
\omega_{b} \approx \omega_{0} \sqrt{1+2 \xi} \approx \omega_{0}(1+\xi)
\end{array} .\right.
$$

Therefore

$$
Q=\frac{1}{2 \xi}=\frac{\omega_{0}}{\omega_{b}-\omega_{a}}
$$

The free vibration equation can be expressed as

$$
\ddot{x}+2 \xi \omega_{0} \dot{x}+\omega_{0}^{2} x=0 \text {. }
$$

By solving this equation, we can get

$$
x(\mathrm{t})=X e^{-\xi \omega_{0} t} \sin \left(\omega_{0} \sqrt{1-\xi^{2}} t+\varphi\right),
$$

where $X$ and $\varphi$ represent the initial amplitude and the initial phase, respectively.

The decay time constant $\tau$ is defined as the time it takes for the initial amplitude to decrease to its $1 / e$ in free vibration, so from function (11) we can get 


$$
\tau=\frac{1}{\xi \omega_{0}} .
$$

Therefore

$$
Q=\frac{1}{2 \xi}=\frac{\omega_{0} \tau}{2} \text {. }
$$

Function (9) and (13) are the basic calculation formulas for $\mathrm{Q}$ factor measurement. In the following paper we denote the former one as the frequency response method and the latter as the ring-down time method.

\section{Measurement system}

\subsection{Resonator structure}

The cylindrical resonator manufactured by our laboratory is made of fused silica [8]. It is composed of four parts: a resonant shell, a vibration-conducting shell, a stem, and a bottom plate, as shown in Fig. 1. Eight circular holes are symmetrically distributed on the bottom plate to reduce the stiffness and the stem is designed to clamp the resonator.

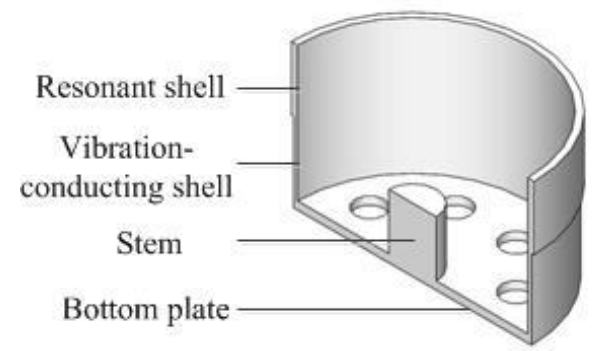

\subsection{Testing system}

Fig. 1 The structure of the cylindrical resonator

With a laser Doppler vibrometer (Polytec, Inc.), rapid non-contact measurement can be achieved. The laser Doppler vibrometry is based on the Doppler effect when light back-scattered from the vibrating resonator surface. As the back-scattered light is modulated by the vibration of the resonator, the frequency of this light changes slightly compared with the incident light. The detector inside the laser Doppler vibrometer then receives the beat signal and the system calculates the corresponding velocity or displacement.

The measurement system is shown in Fig. 2. The resonator is fixed with clamping. The fixture is made of brass with an adjustable holder. The resonator and the fixture are fixed on a rotary table together. The rotary table in this system is used to adjust the excitation angle. The vibration of the resonator is detected by the laser Doppler vibrometer. The whole system is placed on an optical table to decrease the effect of environmental vibration. The measurement is conducted in a super-clean laboratory room with a stable environment.

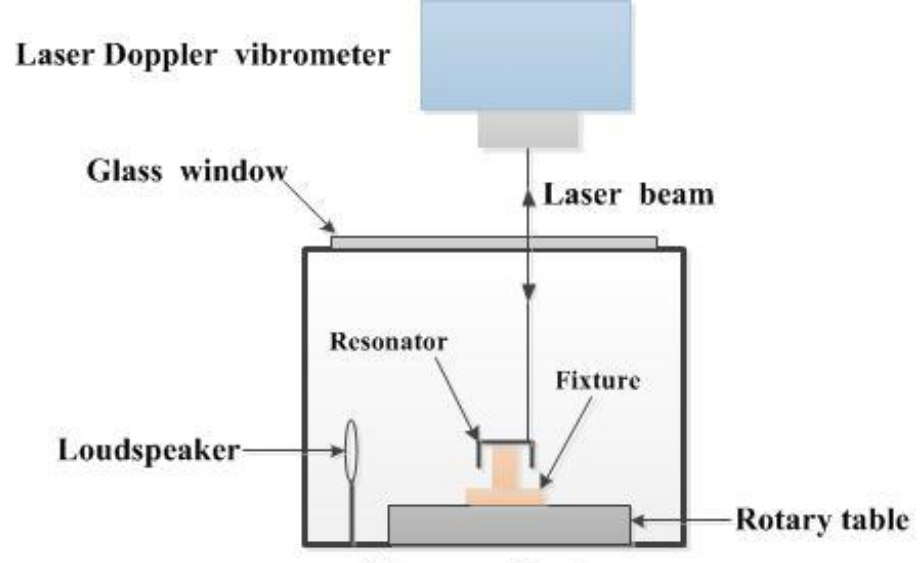

Vacuum chamber

Fig. 2 The set-up of the system. 
Before the measurement, the laser beam should be preferably adjusted to be perpendicular to the bottom plate of the resonator. Then the measurement reference plane is defined by focusing the laser spot on the bottom plate, ensuring that the reference plane coincides with the bottom plate surface. At last, the scanning points are defined on the edge of the bottom plate.

\subsection{Testing procedure}

Based on repeated experiments, the $\mathrm{Q}$ factor measurement procedure is designed as follows:

1. A test point is arbitrary selected from the scanning points. A sweeping signal with a wide frequency range is used to excite the resonator and the laser Doppler vibrometer records the amplitude frequency response signal of the test point, from which we can get the resonant frequency. Then a sweeping signal with a narrow frequency range $(\Delta f= \pm 4 \mathrm{~Hz}$ relative to the rough resonant frequency) is used to get a more accurate frequency $f_{0}$ since a narrower frequency range has a better resolution.

2. A sinusoidal signal with frequency $f_{0}$ is used to excite the resonator. The scanning model of laser Doppler vibrometer is used to record the vibration signal of all points and the vibration model of the resonator is obtained, as shown in Fig. 3.

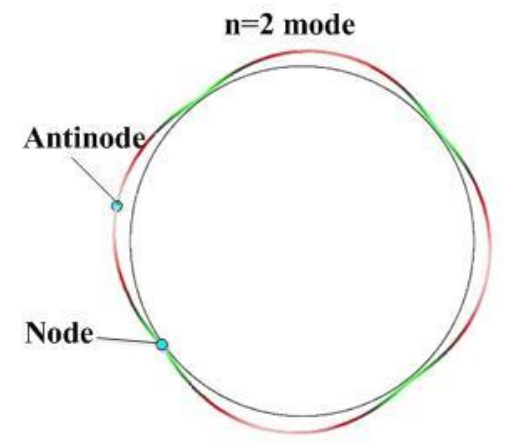

Fig. 3 The wineglass mode of the as-fabricated resonator in air

3. The antinode of the vibration mode is selected as the testing point. A sweeping signal with a frequency range of $\Delta f$ is used to excite the resonator and the time sequence signal of the vibration velocity is recorded by the vibrometer. The FFT transformation of the time sequence signal gives the frequency amplitude response, and the $\mathrm{Q}$ factor of the resonator is then calculated according to Eq. (9), as shown in Fig. 4.

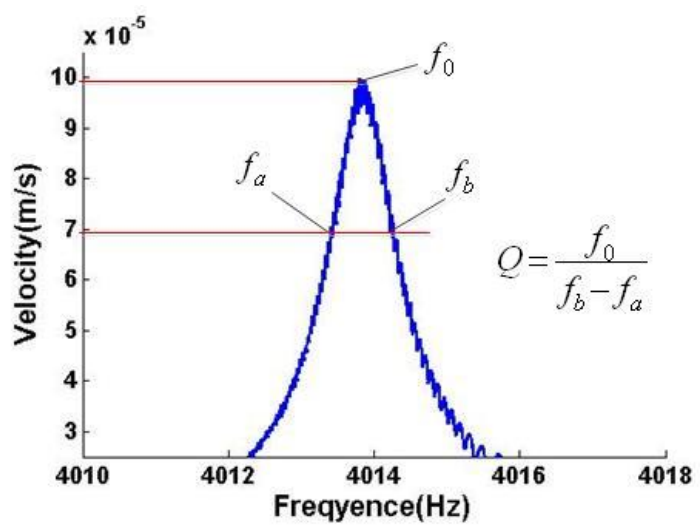

Fig. 4 The frequency response method to calculate $\mathrm{Q}$ factor of the as-fabricated resonator in air

4. With the same testing point in step 3, we record the decay time signal. The resonator is excited by a sinusoidal signal with frequency $f_{0}$. When the vibration velocity is steady in one minute, we shut down the loudspeaker and record the signal by the laser Doppler vibrometer. Then the signal is fitted exponentially in MATLAB and the decay time is calculated, as shown in Fig. 5. The Q factor is calculated using Eq. (13). 


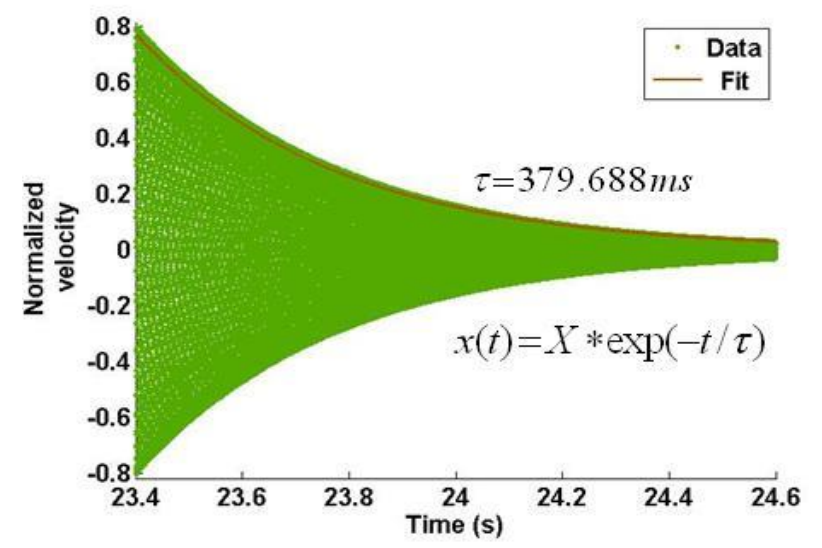

Fig. 5 The ring-down time method to calculate $\mathrm{Q}$ factor of the as-fabricated resonator in air

\section{Results and Discussion}

We used the measurement system introduced in section 3 to measure the Q factor of different resonators in different conditions.

First the $\mathrm{Q}$ factor of an as-fabricated resonator was measured in air, as listed in Table 1.

Table 1 The Q factor of the as-fabricated resonator in air

\begin{tabular}{ccccc}
\hline Testing groups & $\begin{array}{c}\text { Resonant } \\
\text { Frequency } \\
f_{0}[\mathrm{~Hz}]\end{array}$ & $\begin{array}{c}Q \text { factor } \\
\text { calculated by } \\
\text { frequency } \\
\text { response signal }\end{array}$ & $\begin{array}{c}\text { Decay time } \\
\tau \text { [ms }]\end{array}$ & $\begin{array}{c}Q \text { factor } \\
\text { calculated by } \\
\text { decay time }\end{array}$ \\
\hline 1 & 4013.794 & 4732 & 379.688 & 4787 \\
2 & 4013.897 & 4736 & 374.454 & 4721 \\
3 & 4013.928 & 4779 & 377.187 & 4756 \\
4 & 4013.928 & 4828 & 377.930 & 4765 \\
\hline Average & 4013.887 & 4769 & 377.315 & 4757 \\
Standard deviation & 0.0635 & 44.87 & 2.176 & 27.45 \\
Coefficient of variance & $0.00125 \%$ & $0.941 \%$ & $0.576 \%$ & $0.577 \%$ \\
\hline
\end{tabular}

As we can see from Table 1, the resonator frequency is about $4014 \mathrm{~Hz}$. We compared this result with FEM simulation. The parameters of the as-fabricated resonator were used to build the model using ANSYS software, as shown in Fig.6 (a). Modal analysis, as shown in Fig.6 (b) indicated that the frequency of $n=2$ wineglass mode was $4075.2 \mathrm{~Hz}$. The measurement results were close to the simulation results. Meanwhile, the variance coefficients were less than 1\%, suggesting that the vibration parameters measured by this system is repeatable and highly stable. Therefore, we concluded that our $\mathrm{Q}$ factor measurement procedure is reliable. Comparing the $\mathrm{Q}$ factors measured by both methods, the results by the ring-down time method is more stable than that by the frequency response method. Nevertheless, the differences between results measured by each method were within $0.3 \%$.

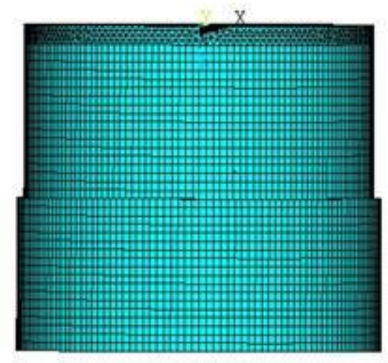

(a)

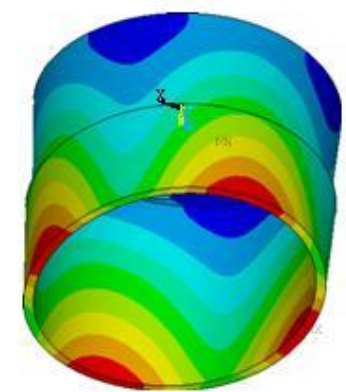

(b)

Fig. 6 The FEM simulation of the as-fabricated resonator. (a) The elevation view of the finite element model. (b) Modal analysis of the $n=2$ wineglass mode. 
In order to explore the versatility of this measurement system, a resonator with post-fabrication surface treatment was measured in air, as listed in Table 2. This resonator was subsequently measured under modest vacuum (about $100 \mathrm{~Pa}$ ), as listed in Table 3.

Table 2 The $\mathrm{Q}$ factor of the resonator with post-fabrication in air

\begin{tabular}{ccccc}
\hline Testing times & $\begin{array}{c}\text { Resonant } \\
\text { frequency } \\
f_{0}[\mathrm{~Hz}]\end{array}$ & $\begin{array}{c}Q \text { factor } \\
\text { calculated by } \\
\text { frequency } \\
\text { response signal }\end{array}$ & $\begin{array}{c}\text { Decay time } \\
\tau \quad[\mathrm{ms}]\end{array}$ & $\begin{array}{c}Q \text { factor } \\
\text { calculated by } \\
\text { decay time }\end{array}$ \\
\hline 1 & 3781.689 & 8860 & 738.555 & 8774 \\
2 & 3781.689 & 8845 & 753.633 & 8953 \\
3 & 3781.665 & 8739 & 747.813 & 8884 \\
4 & 3781.653 & 9085 & 753.633 & 8953 \\
\hline Average & 3781.674 & 8882 & 748.409 & 8891 \\
Standard deviation & 0.0180 & 145.5 & 7.119 & 84.51 \\
Coefficient of variance & $0.000476 \%$ & $1.64 \%$ & $0.951 \%$ & $0.951 \%$ \\
\hline
\end{tabular}

Table 3 The Q factor of the resonator with post-fabrication under low vacuum

\begin{tabular}{ccccc}
\hline Testing times & $\begin{array}{c}\text { Resonant } \\
\text { frequency } \\
f_{0}[\mathrm{~Hz}]\end{array}$ & $\begin{array}{c}Q \text { factor } \\
\text { calculated by } \\
\text { frequency } \\
\text { response signal }\end{array}$ & $\begin{array}{c}\text { Decay time } \\
\tau[\mathrm{ms}]\end{array}$ & $\begin{array}{c}Q \text { factor } \\
\text { calculated by } \\
\text { decay time }\end{array}$ \\
\hline 1 & 3792.047 & 327177 & 32131.41 & 382783 \\
2 & 3792.059 & 336339 & 31397.58 & 374042 \\
3 & 3792.090 & 324571 & 31810.30 & 378962 \\
4 & 3792.114 & 323231 & 31331.72 & 373263 \\
\hline Average & 3792.078 & 327829.5 & 31667.75 & 377263 \\
Standard deviation & 0.0303 & 5904.839 & 374.70 & 4462.13 \\
Coefficient of variance & $0.000799 \%$ & $1.80 \%$ & $1.18 \%$ & $1.18 \%$ \\
\hline
\end{tabular}

The result from Table 2 shows that the $\mathrm{Q}$ factor of the resonator almost doubled in air after the treatment. The coefficients of variance are less than $2 \%$ and the differences of $\mathrm{Q}$ factors measured by two methods are within $0.2 \%$. Results in Table 1 and Table 2 indicate that the frequency response method and the ring-down time method are both reliable when $\mathrm{Q}$ factor is within $10^{4}$ and we can choose the measurement method according to different resonator conditions.

From Table 3, one can see that the coefficients of variance for different parameters are still less than $2 \%$, but the difference of Q factor measured by two methods is more than $13 \%$. In modest vacuum condition, the efficiency of acoustic excitation will decrease due to lower pressure. As a result, the vibration amplitude of the resonator is smaller than that in air. As the testing noise stays constant, the signal to noise ratio in modest vacuum condition is worse than that in air. Since the Q factor is very large, a small change of the $3-\mathrm{dB}$ bandwidth $\left(f_{b}-f_{a}\right)$ will lead to a great variation of $\mathrm{Q}$ factor calculation. However, the ring-down time method avoids this kind of disadvantage. The data from Table 1-3 also shows that the standard deviations of the Q factors by ring-down time method are all smaller than that by frequency response method, which suggests the former method is more stable and reliable than the latter. Therefore, when the Q factor of the resonator is larger than $10^{5}$, the ring-down method will be more accurate.

\section{Conclusions}

This paper presents a non-contact measurement system to measure the $\mathrm{Q}$ factor for the cylindrical resonator, and the testing procedure was carefully designed. Two measurement methods (i.e. the frequency response method and the ring-down time method) were proposed, and the measurement theory and process of both methods were demonstrated. The reliability and stability of two methods were verified by simulations and experiments, and the versatility of the system was 
demonstrated by measuring resonators under different conditions. In conclusion, Q factor from a few thousands to hundreds of thousands can be measured accurately by this system using both methods. For higher $\mathrm{Q}$ factors the ring-down time method is preferred. This study forms the basis of further Q factor research. The method proposed here is simple, intuitive and convenient, which also does not require excessive electronic circuits or extra signal demodulation. Furthermore, it avoids introducing surface damage and additional loss to the resonator. However, further efforts is needed in improving the measurement system, including the design of the fixture to further decrease support loss, better control of the vacuum, optimization of the excitation system, etc.

\section{References}

[1] Rozelle D M. The hemispherical resonator gyro: From wineglass to the planets, C.Proc. 19th AAS/AIAA Space Flight Mechanics Meeting. (2009)1157-1178.

[2] Chikovani V V, Yatsenko Y A, Barabashov A S, et al. Improved accuracy metallic resonator CVG,J. Aerospace and Electronic Systems Magazine, IEEE (2009) 24(5): 40-43

[3] Chikovani V V, Yatzenko Y A, Kovalenko V A. Coriolis force gyroscope with high sensitivity: U.S.Patent7281425.( 2007)

[4] Watson W S. Vibratory gyro skewed pick-off and driver geometry, C. Position Location and Navigation Symposium (PLANS), IEEE/ION. IEEE(2010)171-179.

[5] Xi X, Wu X, Zhang Y, et al. A study on Q factor of the trimmed resonator for vibratory cupped gyroscopes, J. Sensors \& Actuators A Physical, (2014)218(10) 23-32.

[6]Chikovani V V, Okon I M, Barabashov A S, et al. A set of high accuracy low cost metallic resonator CVG, C. Position, Location and Navigation Symposium, IEEE/ION. IEEE(2008)238-243.

[7] Yao P, Dongya W, Yanyan W, et al. Monolithic cylindrical fused silica resonators with high Q factors, Sensors(2016). (Under review)

[8] Ageev A, Palmer B C, Felice A De, et al. Very high quality factor measured in annealed fused silica, J. Classical and Quantum Gravity, (2004)21(16): 3887.

[9] Luan Q, Yulie W, Yi T. Research on Quality Factor of Resonator for Cylinder Shell Vibrating Gyroscope[J]. Chinese Journal of Sensors \& Actuators (2011) 24(10) 1401-1405.

[10] Xi X, Wu X, Wu Y, et al. Structural-acoustic coupling effects on the non-vacuum packaging vibratory cylinder gyroscope, J. Sensors (2013)13(12) 17176-92.

[11] Jeanroy A, Bouvet A, Remillieux G. HRG and marine applications, J. Gyroscopy and Navigation (2014) 5(2) 67-74.

[12] Silva De, Clarence W, ed. Vibration damping, control, and design. CRC Press, 2007. 